\title{
Longer-Term Durability of Using Default Options in the Electronic Health Record to Increase Generic Prescribing Rates
}

\author{
David Olshan, MD ${ }^{1,2}$, Charles A. L. Rareshide, MS ${ }^{7}$, and Mitesh S. Patel, MD, MBA, MS \\ 1, Penn Medicine Nudge Unit, Philadelphia, PA, USA; ${ }^{2}$, Massachusetts General Hospital, Boston, MA, USA; ${ }^{3}$ Perelman School of Medicine, University \\ of Pennsylvania, Philadelphia, PA, USA; ${ }^{4}$ The Wharton School, University of Pennsylvania, Philadelphia, PA, USA; ${ }^{5}$ Crescenz Veterans Affairs Medical \\ Center, University of Pennsylvania, Philadelphia, PA, USA.
}

KEY WORDS: default options; generic medication; physician behavior; durability.

$\mathrm{J}$ Gen Intern Med 34(3):349-50

DOI: $10.1007 / \mathrm{s} 11606-018-4719-9$

(c) Society of General Internal Medicine (This is a U.S. government work and not under copyright protection in the U.S.; foreign copyright protection may apply) 2018

\section{INTRODUCTION}

Default options are the action that takes place if no alternative choice is selected. Default options are abundant within electronic health records (EHRs) and can be used to significantly influence behavior. ${ }^{1}$ For example, we found that changing generic medication prescription settings in the EHR from opt-in to opt-out increased generic prescription rates across our health system from 75 to $98 \%$ in the first 7 months after implementation. ${ }^{2}$ However, the longer-term impact of default options has not been well examined and evidence on the sustainability of other types of nudges for reducing inappropriate antibiotic prescribing has been mixed. ${ }^{3,4}$ The objective of this study was to evaluate the 2.5-year durability of the increase in generic prescribing rates after changing EHR prescription default settings.

\section{METHODS}

Similar to prior work, ${ }^{2}$ we compared monthly generic prescription rates from January 2014 to May 2017 for all outpatient clinics at Penn Medicine for oral medications often prescribed for nine common medical conditions including acid reflux, anxiety and/or insomnia, bacterial infection, depression, diabetes, hyperlipidemia, hypertension and/or congestive heart failure, hypothyroidism, and pain. Medications were excluded if they did not have a generic equivalent available during the entire study period, were combination pills, were refills of an existing medication, or were prescribed at our health system on average less than 100 times per month. Data was obtained from Clarity, an Epic reporting database. Generic prescribing rates were compared between the pre-intervention period (January 2014 to October 2014) and the postintervention period (December 2014 to May 2017) using $\chi^{2}$ tests. We used a significance level of $P<.05$ and hypothesis

Published online October 30, 2018 tests were two-sided. This study was approved by the University of Pennsylvania's institutional review board.

\section{RESULTS}

The sample comprised 76 medications. During the 10-month pre-intervention period, $75.4 \%(542,961 / 720,318)$ of prescriptions were generic. During the 30 -month post-intervention period, $98.4 \%(2,558,039 / 2,599,045)$ of prescriptions were generic $(P<.001$ compared to pre-intervention period). Figure 1 displays monthly prescriptions rates which were mostly flat during the post-intervention period. Levothyroxine was an outlier with monthly generic prescription rates in the postintervention period ranging from 76.1 to $80.8 \%$.

\section{DISCUSSION}

Our study has two main findings. First, overall increases in generic prescription rates across the health system were sustained 2.5 years after implementation of an opt-out generic prescribing system within the EHR. In the USA, generic medications are still often under prescribed leading to unnecessary cost and lower adherence. ${ }^{5}$ Health systems may be able to use default options to change clinician prescribing behaviors over longer-term periods. Second, clinicians opted-out of generic levothyroxine for the brand name prescription 20 $25 \%$ of the time. This confirms findings from our prior work ${ }^{2}$ in which clinicians recognize that generic and brand versions of this medication may differ in formulation, and that patients coming to our health system already on the brand formulation should remain on it. This demonstrates that clinician autonomy in medical decision-making is maintained and that opt out rates were constant over this longer-term period.

Our study is limited to one health system and data on prescription orders. Some brand name prescriptions could have been substituted to generic at the pharmacy. However, evidence indicates that substantial excess spending on brand name prescriptions are still occurs. ${ }^{5}$ Our study is also limited to only new prescriptions and default settings for refilled prescriptions were not changed.

To our knowledge, this is one of the longest follow-up evaluations of the impact of nudges on clinician behavior. 


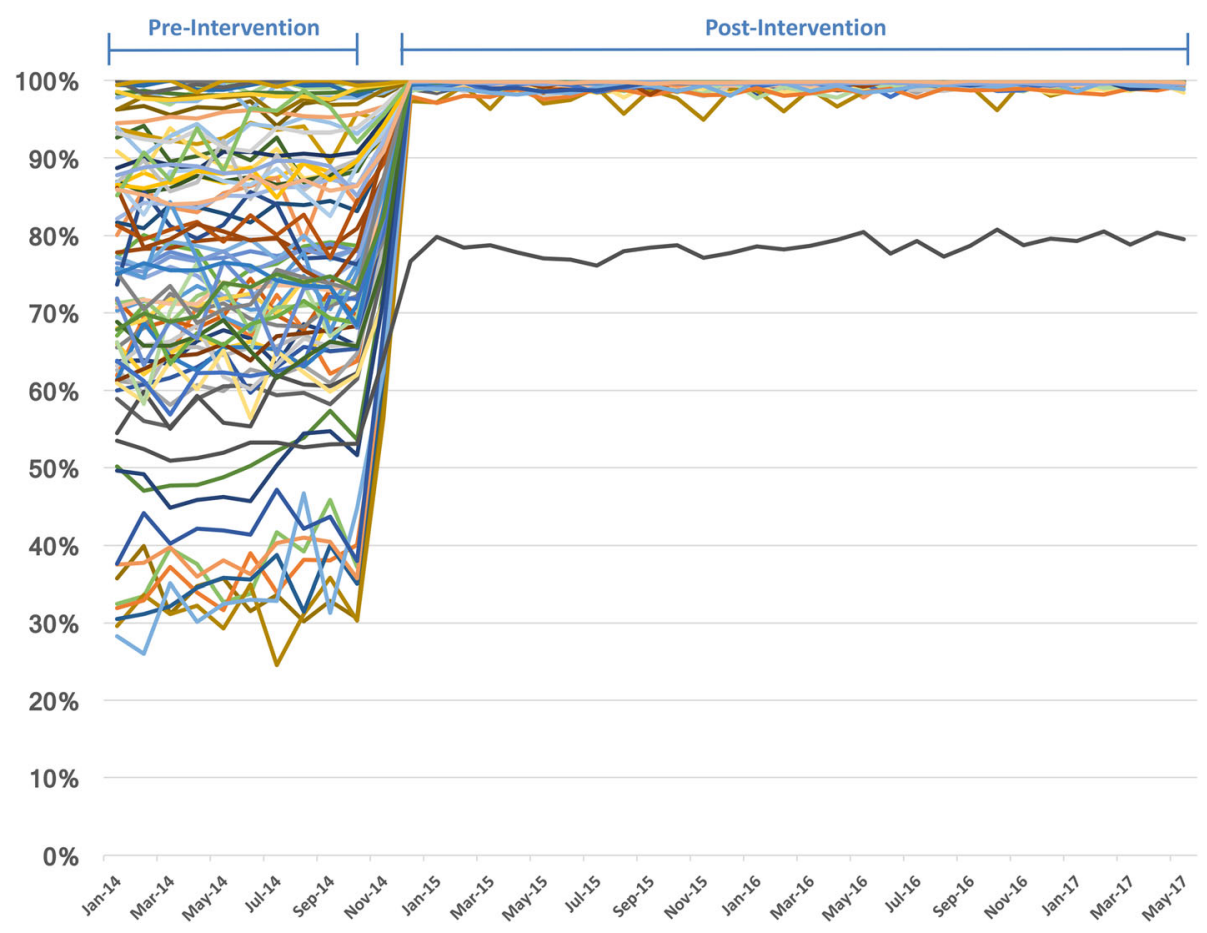

Figure 1 Generic prescription rates for each medications by month. Each line represents one of the 76 medications evaluated in this study. Preintervention period was from January to October 2014. Post-intervention period was from November 2014 to May 2017. Black line ranging from 76.1 to $80.8 \%$ in the post-intervention period represents generic prescription rate for levothyroxine.

Our findings indicate that changing default options in the electronic health record can lead to sustained changes in clinician behavior.

Funding/Support. This study was funded by the University of Pennsylvania Health System through the Penn Medicine Nudge Unit.

Corresponding Author: Mitesh S. Patel, MD, MBA, MS; Crescenz Veterans Affairs Medical Center, University of Pennsylvania, Philadelphia, PA, USA (e-mail: mpatel@upenn.edu).

Authors' Contribution Drs. Olshan and Patel had full access to all the data in the study and take responsibility for the integrity of the data and the accuracy of the data analysis.

\section{Compliance with Ethical Standards:}

Conflict of Interest: Dr. Patel is supported by career development awards from the Department of Veterans Affairs HSR\&D and the Doris Duke Charitable Foundation. Dr. Patel is founder of Catalyst Health, a technology and behavior change consulting firm. Dr. Patel also has received research funding from Deloitte, which is not related to the work described in this manuscript. All remaining authors declare that they do not have a conflict of interest.

Role of the Funder/Supporter: The funding source had no role in the design and conduct of the study; collection, management, analysis, and interpretation of the data; preparation, review, or approval of the manuscript; and decision to submit the manuscript for publication.

\section{REFERENCES}

1. Patel MS, Volpp KG, Asch DA. Nudge Units to Improve the Delivery of Health Care. N Engl J Med. 2018;378(3):214-216.

2. Patel MS, Day SC, Halpern SD, et al. Generic Medication Prescription Rates After Health System-Wide Redesign of Default Options Within the Electronic Health Record. JAMA Intern Med. 2016;176(6):847-848.

3. Gerber JS, Prasad PA, Fiks AG, et al. Durability of benefits of an outpatient antimicrobial stewardship intervention after discontinuation of audit and feedback. JAMA. 2014;312(23):2569-2570.

4. Linder JA, Meeker D, Fox CR, et al. Effects of Behavioral Interventions on Inappropriate Antibiotic Prescribing in Primary Care 12 Months After Stopping Interventions. JAMA. 2017;318(14):1391-1392.

5. Choudhry NK, Denberg TD, Qaseem A, Clinical Guidelines Committee of American College of P. Improving Adherence to Therapy and Clinical Outcomes While Containing Costs: Opportunities From the Greater Use of Generic Medications: Best Practice Advice From the Clinical Guidelines Committee of the American College of Physicians. Ann Intern Med. 2016;164(1):41-49. 